\title{
A Five-Step Strategy to Combine Data Sources from Multiple Wearable Sensors
}

\author{
James A. Levine ${ }^{1,2}$ \\ ${ }^{1}$ Obesity Solutions, Mayo Clinic, Scottsdale, AZ, USA \\ ${ }^{2}$ Obesity Solutions, Arizona State University, Tempe, AZ, USA \\ Email: Levine.james@mayo.edu
}

How to cite this paper: Levine, J.A. (2017) A Five-Step Strategy to Combine Data Sources from Multiple Wearable Sensors. Technology and Investment, 8, 33-43. https://doi.org/10.4236/ti.2017.81003

Received: December 31, 2016

Accepted: February 1, 2017

Published: February 4, 2017

Copyright (c) 2017 by author and Scientific Research Publishing Inc. This work is licensed under the Creative Commons Attribution International License (CC BY 4.0).

http://creativecommons.org/licenses/by/4.0/

\begin{abstract}
With the multitude of non-communicating wearable sensors, there is an urgent need to better combine wearable data streams in order to improve human health and well-being. A five-step process is proposed. The first step is to specify the human behavior that the data set will address. The second step is to critically assess primary measurement that allows the behavioral goal to be addressed. After this, other streams can be integrated in a hierarchical fashion based on their accuracy, precision and relevance. The third step is to perform a hierarchical synthesis of the multiple data streams. In the fourth step, the multiple data streams are integrated for practical use; we propose achieving this with wearable computers. The final step is that system retraining occurs, via Artificial Intelligence, so that an integrated wearable system can be individualized. A case study of Type 1 diabetes is used: this analysis and the proposed solutions illustrate the need for an urgent interdisciplinary debate to advance useful methods for combining data from divergent wearable sensors. Wearable fully integrated systems, programmed with Artificial Intelligence, will enable data from multiple wearable sensors to be optimized to improve individual well-being.
\end{abstract}

\section{Keywords}

Wearable Technology, Consumer Behavior, Entrepreneurship, Investment, Artificial Intelligence

\section{Introduction: The Wearable Sensor Market Is Growing}

The $\$ 1$ billion wearable sensor market is predicted to grow ten-fold over the next five years [1]. The wearable sensor market is growing for a variety of reasons [2]. Firstly, there is increasing consumer demand and availability of wearable sensors. Secondly, there is a growing acceptability for wearable sensor use in society. 
The most common of these are wearable sensors that monitor daily physical activity levels [3]. However, other sensors are gaining popularity such as the monitor sleep, intradermal variables and heart rate [4]. Thirdly, the cost of developing mass scalable electronics is declining, so the barrier to producing new compact wearable technologies has diminished. Fourthly, there is a growing awareness that the Internet of Things [5] will be important in society. This has propagated the advancement of big data analyses and the systems to perform these analyses [6] [7]. The wearable sensor market is expanding and it will be necessary to better integrate non-communicating wearable technologies to improve human well-being.

\section{Wearable Sensors Generate Data That Vary in Format, Quality and Integrity in the Absence of a Common Analytical Approach}

Wearable sensors have the capability of gathering data from different physiological variables and so could improve human well-being [2]. Data may be gathered using different technological modalities that may produce different types and quality of data, of variable value and validity [8]. Data streams may be even more heterogeneous because different companies produce sensors under variable stringencies and governed by different regulatory rule sets; for example in the U.S., subcutaneous continuous glucose monitoring requires FDA approval whereas monitoring daily movement requires no regulatory oversight [9]. Methods for analysis vary too; some data are filtered and analyzed on board the device whereas other devices stream raw data to a secondary data acquisition system [8] [9]. The quality, integrity and nature of different wearable sensors vary depending upon a multitude of factors.

\section{Once Data are Gathered, the Applications of the Data are Multidimensional and Inconsistent}

Some data, such as blood sugar data, may need to be accessed by both the user and a third party, such as a healthcare provider [9]. Data from movementtrackers, by contrast, is generally only accessed by users themselves for selfmonitoring [3]. Despite data from diverse wearable sensors being of different types and applications, uniform safeguards are necessary [7]. Data integrity needs to be guaranteed, data particularly if health-related, needs to be private and secure and data transmission protocols need to be robust [6] [7].

\section{The Disorganization of Data Handling in the Wearable Sensor Field Makes Intelligent Analysis and Consistent Feedback Impossible}

Because the entire process of data acquisition, transmission and analysis from wearable sensors is unfettered, it is impossible to integrate data for individual or third party use [8]. If airlines communicated with airports in a haphazard fashion, without agreed upon protocols, chaos would reign. Regardless of the 
challenge of terminal integration of multi-stranded data from wearable sensors, there have been few structured approaches to standardizing the data from wearable sensors and intelligently unifying data integration, analysis and feedback.

\section{The Multiplicity of Wearable Sensors}

Many wearable sensors exist and others are being developed for human use [2]. The wearable sensor field is at a critical juncture, because common approaches to integrating, analyzing and processing the multiple sources of data has not occurred. If people are to benefit, common approaches to data transmission, integration and processing will be needed.

\section{Strategy to Build an Intelligent Multisensor System}

In order to work through the complexity of how to integrate multiple disparate sensors it is necessary to develop a robust strategic approach. There are five steps that can be taken to integrate data from multiple wearables:

Step 1. Identify a specific problem that the data set will address

Step 2. Identify a hierarchy of data streams from wearable sensors:

1) Define a valid primary measurement to verify true events and exclude false alarms;

2) Define secondary measures in hierarchical order that contribute to the causation of true events and/or exclusion of false alarms:

a) Define the secondary measure with the highest hierarchical value that contributes to causation of true events and/or exclusion of false alarms;

b) Define the secondary measure with the second highest hierarchical value that contributes to causation of true events and/or exclusion of false alarms;

c) Define a secondary measure-with the third highest hierarchical value that contributes to causation of true events and/or exclusion of false alarms etc.

Step 3. Hierarchical Data synthesis

Synthesize data based upon a clearly defined problem that needs to be solved with an evidence-based understanding of the hierarchical value of the data sources.

\section{Step 4. Practical integration of data streams}

The data streams need to be integrated in a practical fashion that free-living people can apply outputs to their health and/or behaviors.

Step 5. System Retraining to achieve individualization

Re-training of the system needs to occur based on the presence of false positive and false negative events. Artificial Intelligence approaches can individualize the system's algorithms.

\section{Type I Diabetes Is a Chronic Disease That Serves as an Example of How Important It Is to Integrate Data from Multiple Wearable Sensors}

There are a variety of reasons as to why Type I (Juvenile) diabetes is a valuable example of a chronic disease state by which we can examine the challenges and 
potential solutions as to how data from multiple disparate wearable sensors can be integrated [9] [10] [11] [12] [13].

In Type I diabetes there is autoimmune destruction of the insulin producing cells in the pancreas [14]. Patients, often diagnosed in childhood, have lifelong dependency on exogenous insulin. Multiple aspects of a patient's life affect insulin dosage; for example, if a patient overexerts him or herself without decreasing their insulin dose, they can develop low blood sugar values-hypoglycemia [15]. Hypoglycemia is a major cause of sudden death in patients with Type 1 diabetes [16] [17]. Conversely, if a patient eats too much their insulin dose may be inadequate and they become hyperglycemic (high blood glucose) [14]. It is known through multicenter trials that good control of blood glucose is associated with improved outcomes in type I patients [15] [18]. Therefore there is a lifelong need to maintain good glycemic control. Type I diabetes is a chronic disease state where wearable sensors could be useful because there are many aspects of daily living that can impact their activity level and food intake [9].

\subsection{Identification of a Specific Human Problem}

We will examine the following clinical scenario: A physician is referred a 36year-old woman with Type 1 diabetes because whilst at work she has multiple hospital admissions with low blood sugar-hypoglycemia (Figure 1. Panel A). Episodes of hypoglycemia are the commonest causes of acute harm to patients with Type 1 diabetes [15]. They can be caused by excessive insulin and/or inadequate caloric intake for the physiological state of the patient [16] [17].

At the time of her evaluation the patient had lived with Type 1 diabetes for 23 years. She checked her blood glucose periodically with a lancet and adjusted her insulin injections accordingly. The doctor, on questioning the patient, discovered that the hypoglycemia events occurred only in the afternoons. The patient explained that in the afternoons she often had to hand deliver documents around the work campus. The doctor hypothesized that the low blood sugar events occurred because of high levels of physical exertion.

\subsection{Primary Measurement Verifies That the Problem Exists}

To verify that the patient indeed had low blood sugar values in the afternoons, the doctor fitted the patient with a wearable glucose monitor that measured the patient's glucose continuously and aggregated a value every 15 minutes [19] [20]. The patient's 15-minutely analog glucose data were converted to a digital signal (Figure 1. Panel B): When the patient had a glucose value that was high $(>150 \mathrm{mg} / \mathrm{dL}), a+1$ signal was generated. When the patient had a glucose value that was low $(<90 \mathrm{mg} / \mathrm{dL})$ a -1 signal was generated. All other glucose values were ascribed, ' 0 '. This digital signal was transmitted to the doctor as a series of $+1,-1$ and 0 digits (Figure 1. Panel E). The data confirmed that the low blood sugar values occurred in the afternoon (Figure 1. Panel B). 
A. IDENTIFICATION OF A SPECIFIC PROBLEM The 'human' problem: a 36 y/o woman with Type 1 diabetes has several low blood sugar values at work requiring hospitalization

B. SENSOR 1: PRIMARY MEASUREMENT VERIFIES THAT THE PROBLEM EXISTS. Person wears continuous subcutaneous blood sugar sensor. Red arrows confirm the problem exists

\section{SENSOR 2: SENSOR TO ADDRESS THE} HYPOTHESIS. (High physical activity?). Person wears continuous physical activity sensor. Green arrows support causality

\section{SENSOR 3: SENSOR TO ADDRESS A} SECONDARY/SUBSIDIARY HYPOTHESIS. (High ambient temperature?). Continuous wearable ambient temperature sensor. Green narrows confirm minor impact of high temperature

6am noon $4 \mathrm{pm}$ midnight
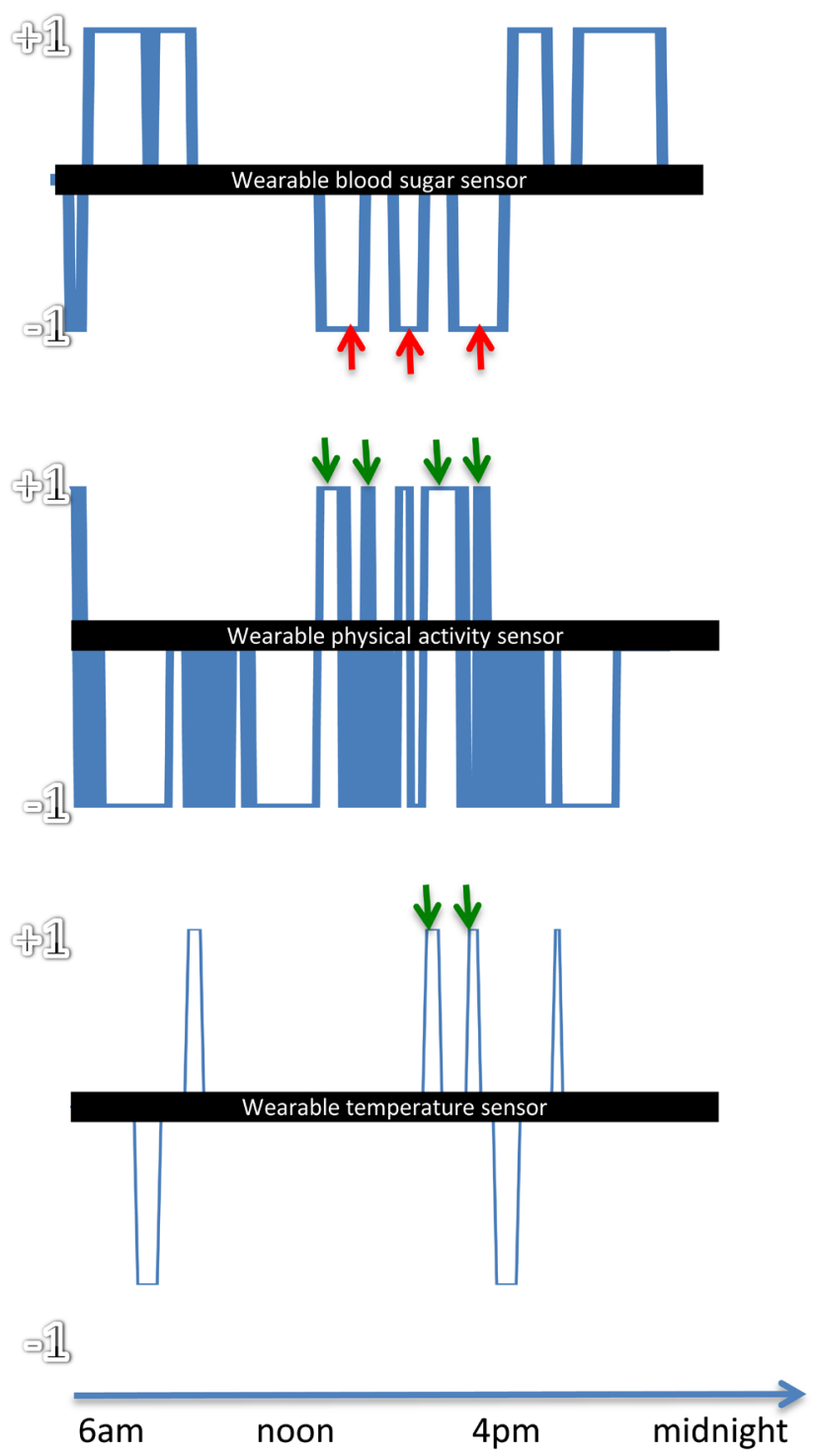

E. SYNTHESIS: Sample data set.

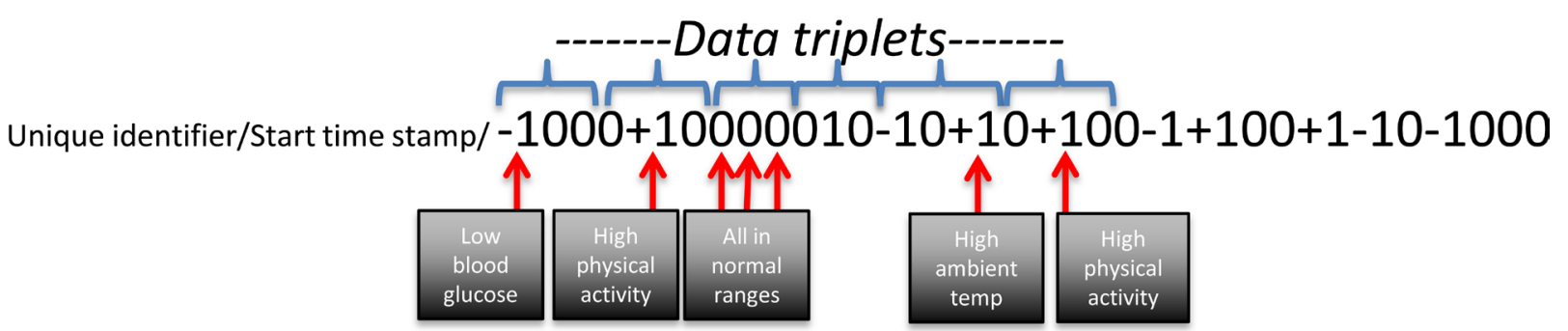

Figure 1. Worked example of Type 1 diabetes and the integration of three wearable sensors. 


\subsection{Identify Sensor/s to Address the Primary Hypothesis}

To address the hypothesis that, "low blood sugar events occurred because of high levels of physical exertion", the doctor asked the patient wear a motion sensor to capture the patient's daily physical activity. The patient's daily physical activity values ranges from 6 to 22 Activity Units (AU)/min with a mean of 14 and standard deviation of $4 \mathrm{AU} / \mathrm{min}$ [8] [13]. The analog-to-digital converter for this sensor was set as follows; +1 was sent for $\geq 19 \mathrm{AU} / \mathrm{min},-1$ was sent $\leq 9$ $\mathrm{AU} / \mathrm{min}$ and all other values were assigned " 0 ". When the doctor (or algorithm) reviewed the outputs from the glucose monitor and the activity monitor side-byside, +1 elevations in physical activity coincided with -1 depressions in blood glucose (Figure 1. Panels B and C). The doctor's hypothesis was confirmed, that low blood sugar events occurred when the patient was physically exerting herself by hand-rushing documents across her work campus.

\subsection{Identify sensor/s to Address a Secondary/Subsidiary Hypothesis}

The patient improved by taking a larger snack before hand-rushing documents around her campus. She had far fewer low blood sugar events. However, during the summer, the doctor noticed that the patient was getting more low blood sugar values requiring treatment. The doctor hypothesized (secondary hypothesis) that the patient was getting more, low blood sugar events because of extremely high summer temperatures. The patient was asked to wear an ambient temperature sensor (Sensor 3) attached to the top of her glasses. A +1 signal was sent when ambient temperature increased by $3^{\circ} \mathrm{C}$ above the rolling average for 12 hours previously, and a -1 one signal when ambient temperature decreased by $3^{\circ} \mathrm{C}$; all other outputs were 0 . When the 3 digital signals were viewed alongside each other (Figure 1. Panels B, C, D), low blood sugars were influenced by high ambient temperature. The patient was advised to stay hydrated on summer afternoons at work.

\subsection{Hierarchical Data Synthesis}

In this example, three signals from three divergent wearable sensors were combined using an analog-to-digital conversion approach. The data format from the three sensors was different (glucose, movement and temperature), the data-gathering frequencies were different and data integrity was different. In the patient described above with Type 1 diabetes:

- All 3 sets of data were transmitted together (Figure 1. Panel E) using a format that rendered the information unidentifiable, codified (without knowing the sensor configuration and the rules for $+1 /-1 / 0$ ) and so secure.

- The data were intelligently integrated to improve health.

- Data of different veracity were not equally weighted. The driving data set (Sensor 1: blood glucose values) were of predominant importance because they were being used to drive the principal question. Sensor 2 addressed the primary hypothesis and so had secondary weighting. Sensor 3 addressed a 
subsidiary hypothesis and so had tertiary weighting.

- The analogue-to-digital conversion described above illustrates an example of how variable data from multiple wearable sensors can be usefully combined.

The three wearable sensors in this example address a specific health problem. Exactly the same five-step approach can be used to address health questions for a person wearing 15 different sensors (Figure 2). Also, multiple different health questions can be addressed using the same sensor streams but with different prioritization and weighting.

- The common (digitized) data format is the key starting point to integrate data from different sensors.

- This approach is consistent with multiple companies simultaneously developing different sensors with different outputs (all outputs in this example were reduced to: $+1 /-1 / 0$ format).

- This approach enables disparate and complex data sources to be transmitted in a de-identified and secure fashion.

- This approach enables intelligent design to drive the process for using wearable sensors to advance human welfare.

This approach for combining data from multiple wearable sensors is one simple method for combining data from multiple divergent sources. It was used here only to illustrate the challenges. Many other mathematical approaches can be used to intergrade medical signals. For example Fourier transformations are relevant in a broad series of chronic disease states such as, Alzheimer's disease, cholelithiasis and breast cancer [21]-[26]. Similarly, the Gaussian function has been applied to cerebral ischemia, rheumatoid arthritis and chemotherapy efficacy in cancer treatment [27] [28] [29] [30].

Regardless of the mathematical paradigm/s being applied to multiple streams of data, an organized and hypothesis driven approach is recommended.

\subsection{Data Integration (Figure 2)}

Although the need for integrated wearable technologies in health is discussed [4], practical solutions are rarely expounded upon. However, relatively simple solutions do exist for integrating data from multiple wearable sensors with different data formats. For example, the Raspberry Pi 3

(https://www.raspberrypi.org/products/raspberry-pi-3-model-b/) [31]. The computer measures $86 \times 17 \times 54 \mathrm{~mm}$ and weighs $42 \mathrm{~g}$. The system runs Linux and enables multiple inputs with high-level data processing under the ARMv8 (1.2 $\mathrm{GHz}$ 64-bit quad-core) processor. The Pi 3 computer has both Wi-Fi (802.11n) and Bluetooth (4.1 and Low Energy) connectivity. There are 4 USB ports, 40 GPIO pins, and a Micro SD slot. Coin cell batteries power the unit, enabling continuous use for 20 days.

Practically, complex algorithms can be written in Linux so that complex analytics can run on the fly. In medical care scenarios, such systems can communicate with the medical teams in real time, potentially using the cell phone network, to ensure medical oversight (Figure 2). It is envisaged that a wearable 


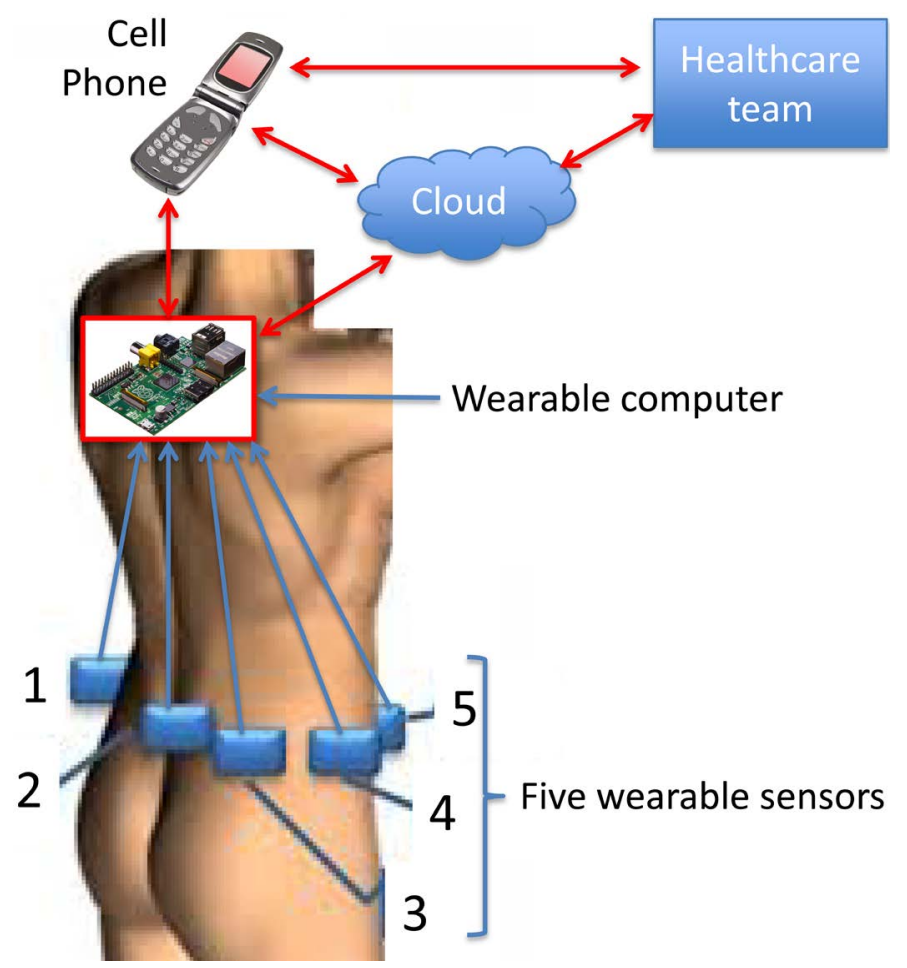

Figure 2. Example of how a multiple-sensor system can be practically integrated using a wearable computer that has WiFi and Bluetooth capability.

computer could integrate multiple inputs for multiple wearable sensors simultaneously. The Pi3 can drive outputs too, such as a personalized warning signal (e.g. "Your blood sugar is decreasing, eat something now!") or even drug delivery [32]. Integrated signals can be used to improve personal behaviors or health [33].

\subsection{System Training}

Artificial intelligence (AI) is in its infancy. Using a variety of metadata learning tools such as multi-layer perceptron neural network learning, it will be possible for multifaceted systems to improve their data analysis autonomously [34] [35] [36]. This approach is already being used to help patients with epilepsy, analyze EKGs and work out how to optimize the integration of genetic data with phenotype [34] [35] [37] [38]. AI is particularly relevant for integrating wearables because these types of complex analytics are necessary to improve true positive rates and decreases false negative rates; reflective of improved positive predictive power.

\section{Conclusion}

This paper illustrates the need for an urgent interdisciplinary debate to advance useful methods for combining data from divergent wearable sensors to help people. Wearable fully integrated systems, programmed with Artificial Intelligence, will enable data from multiple wearable sensors to be optimized for the individual. Integrated wearable systems may assist in the care of patients with chronic diseases such diabetes, cardiac and neurological conditions. Thoughtful integration of wearable sensors has the potential to improve the well-being and health of many people. 


\section{Acknowledgements}

This work was funded by NIH grants, R42 DK085738-02, 5R01DK085516-05 and R015R01HL114024-1 and a CODE Award from Mayo Clinic Center for Innovation.

\section{References}

[1] Levine, J.A. (2016) The Baetylus Theorem-The Central Disconnect Driving Consumer Behavior and Investment Returns in Wearable Technologies. Technology Investments, 7, 59-65. https://doi.org/10.4236/ti.2016.73008

[2] Bandodkar, A.J. and Wang, J. (2014) Non-Invasive Wearable Electrochemical Sensors: A Review. Trends in Biotechnology, 32, 363-371. https://doi.org/10.1016/j.tibtech.2014.04.005

[3] Attal, F., et al. (2015) Physical Human Activity Recognition Using Wearable Sensors. Sensors (Basel), 15, 31314-31338. https://doi.org/10.3390/s151229858

[4] Linden, M. and Bjorkman, M. (2014) Embedded Sensor Systems for Health- Providing the Tools in Future Healthcare. Studies in Health Technology and Informatics, 200, 161-163.

[5] Miranda, J., et al. (2016) An Open Platform for Seamless Sensor Support in Healthcare for the Internet of Things. Sensors (Basel), 16, 2089.

https://doi.org/10.3390/s16122089

[6] Mann, R.P., et al. (2016) The Problem with Big Data: Operating on Smaller Datasets to Bridge the Implementation Gap. Front Public Health, 4, 248.

https://doi.org/10.3389/fpubh.2016.00248

[7] Schaeffer, C., et al. (2016) Big Data Management in US Hospitals: Benefits and Barriers. Proceedings of the Business and Health Administration Association Annual Conference, Chicago.

[8] Gao, W., et al. (2016) Fully Integrated Wearable Sensor Arrays for Multiplexed in Situ Perspiration Analysis. Nature, 529, 509-514.

https://doi.org/10.1038/nature16521

[9] Heintzman, N.D. (2015) A Digital Ecosystem of Diabetes Data and Technology: Services, Systems, and Tools Enabled by Wearables, Sensors, and Apps. Journal of Diabetes Science and Technology, 10, 35-41. https://doi.org/10.1177/1932296815622453

[10] Drion, I., et al. (2015) The Effects of a Mobile Phone Application on Quality of Life in Patients With Type 1 Diabetes Mellitus: A Randomized Controlled Trial. Journal of Diabetes Science and Technology, 9, 1086-1091. https://doi.org/10.1177/1932296815585871

[11] Manohar, C., et al. (2013) Comparison of Physical Activity Sensors and Heart Rate Monitoring for Real-Time Activity Detection in Type 1 Diabetes and Control Subjects. Diabetes Technology \& Therapeutics, 15, 751-757. https://doi.org/10.1089/dia.2013.0044

[12] Montori, V.M., et al. (2004) Telecare for Patients with Type 1 Diabetes and Inadequate Glycemic Control: A Randomized Controlled Trial and Meta-Analysis. Diabetes Care, 27, 1088-1094. https://doi.org/10.2337/diacare.27.5.1088

[13] Zecchin, C., et al. (2013) Physical Activity Measured by Physical Activity Monitoring System Correlates with Glucose Trends Reconstructed from Continuous Glucose Monitoring. Diabetes Technology \& Therapeutics, 15, 836-844.

https://doi.org/10.1089/dia.2013.0105 
[14] Hinshaw, L., et al. (2013) Diurnal Pattern of Insulin Action in Type 1 Diabetes: Implications for a Closed-Loop System. Diabetes, 62, 2223-2239. https://doi.org/10.2337/db12-1759

[15] Schultz, A.T. and Smaldone, A. (2016) Components of Interventions That Improve Transitions to Adult Care for Adolescents with Type 1 Diabetes. Journal of Adolescent Health, 60, 133-146. https://doi.org/10.1016/j.jadohealth.2016.10.002

[16] Gandhi, G.Y., et al. (2011) Efficacy of Continuous Glucose Monitoring in Improving Glycemic Control and Reducing Hypoglycemia: A Systematic Review and Meta-Analysis of Randomized Trials. Journal of Diabetes Science and Technology, 5, 952-965. https://doi.org/10.1177/193229681100500419

[17] Parsaik, A.K., et al. (2012) Population-Based Study of Severe Hypoglycemia Requiring Emergency Medical Service Assistance Reveals Unique Findings. Journal of Diabetes Science and Technology, 6, 65-73.

https://doi.org/10.1177/193229681200600109

[18] Viana, L.V., et al. (2016) Interventions to Improve Patients' Compliance with Therapies Aimed at Lowering Glycated Hemoglobin (HbAlc) in Type 1 Diabetes: Systematic Review and Meta-Analyses of Randomized Controlled Clinical Trials of Psychological, Telecare, and Educational Interventions. Trials, 17, 94.

https://doi.org/10.1186/s13063-016-1207-6

[19] Kuehn, B.M. (2016) Wearable Biosensors Studied for Clinical Monitoring and Treatment. JAMA, 316, 255-257. https://doi.org/10.1001/jama.2016.6240

[20] Seo, D., Paek, S., Oh, S., Seo, S. and Paek, S. (2016) A Human Serum-Based Enzyme-Free Continuous Glucose Monitoring Technique Using a Needle-Type BioLayer Interference Sensor. Sensors, 16, 1581. https://doi.org/10.3390/s16101581

[21] Cheheltani, R., et al. (2016) Fourier Transform Infrared Spectroscopic ImagingDerived Collagen Content and Maturity Correlates with Stress in the Aortic Wall of Abdominal Aortic Aneurysm Patients. Cardiovascular Engineering and Technolo$g y, 1-11$.

[22] Jain, U., Singh, A., Kuchhal, N. and Chauhan, N. (2016) Glycated Hemoglobin Biosensing Integration Formed on Au Nanoparticle-Dotted Tubular $\mathrm{TiO}_{2}$ Nanoarray. Analytica Chimica Acta, 945, 67-74. https://doi.org/10.1016/j.aca.2016.09.026

[23] Lou, S., et al. (2016) Prognostic Metabolite Biomarkers for Soft Tissue Sarcomas Discovered by Mass Spectrometry Imaging. Journal of the American Society for Mass Spectrometry, 28, 376-383. https://doi.org/10.1007/s13361-016-1544-4

[24] Mandel, N.S., Mandel, I.C. and Kolbach-Mandel, A.M. (2016) Accurate Stone Analysis: The Impact on Disease Diagnosis and Treatment. Urolithiasis, Online.

[25] Mordechai, S., Shufan, E., Porat Katzc, B.S. and Salman, A. (2016) Early Diagnosis of Alzheimer's Disease Using Infrared Spectroscopy of Isolated Blood Samples Followed by Multivariate Analyses. Analyst, Online.

[26] Mubtasim, N., Kabir, E., Podder, A. and Bhadra, S. (2016) A Pragmatic Approach to the Analysis of a Combination Formulation. Saudi Pharmaceutical Journal, 24, 689697. https://doi.org/10.1016/j.jsps.2015.06.004

[27] Chapman, J.W., et al. (2011) Comparison of Innovative Estimation of Efficacy to Standard Using the ACCENT Database. Journal of Clinical Oncology, 29, 3616.

[28] Coombes, R.C., et al. (2011) An Open-Label Positron Emission Tomography (PET) Study to Investigate and Quantify Brain and Tumor Penetration of Carbon-11 Labeled Lapatinib in Patients with HER2-Overexpressing (HER2+) Advanced or Metastatic Breast Cancer (MBC). Journal of Clinical Oncology, 29, 107.

[29] Curtis, J.R., et al. (2016) The Clinical Status and Economic Savings Associated with 
Remission among Patients with Rheumatoid Arthritis: Leveraging Linked Registry and Claims Data for Synergistic Insights. Pharmacoepidemiology and Drug Safety, 1-10. https://doi.org/10.1002/pds.4126

[30] Li, Q., et al. (2016) Dynamic Mislocalizations of Nuclear Pore Complex Proteins after Focal Cerebral Ischemia in Rat. Journal of Neuroscience Research, Online.

[31] Katta, M.A., Khan, A.W., Doddamani, D., Thudi, M. and Varshney, R.K. (2015) NGS-QCbox and Raspberry for Parallel, Automated and Rapid Quality Control Analysis of Large-Scale Next Generation Sequencing (Illumina) Data. PLoS ONE, 10, e0139868. https://doi.org/10.1371/journal.pone.0139868

[32] Floares, A.G., et al. (2004) Optimal Drug Dosage Regimens in Cancer Chemotherapy with Neural Networks. Journal of Clinical Oncology, 22, 2134.

[33] Karp, P.D. (2016) Can We Replace Curation with Information Extraction Software? Database, Online.

[34] Ahmad, F., Ashidi Mat Isa, N., Hussain, Z. and Osman, M. (2013) Intelligent Medical Disease Diagnosis Using Improved Hybrid Genetic Algorithm-Multilayer Perceptron Network. Journal of Medical Systems, 37, 9934. https://doi.org/10.1007/s10916-013-9934-7

[35] Aslan, K., Bozdemir, H., Sahin, C. and Noyan Ogulata, S. (2010) Can Neural Network Able to Estimate the Prognosis of Epilepsy Patients According to Risk Factors? Journal of Medical Systems, 34, 541-550.

https://doi.org/10.1007/s10916-009-9267-8

[36] deVries, S.I., et al. (2011) Evaluation of Neural Networks to Identify Types of Activity Using Accelerometers. Medicine \& Science in Sports \& Exercise, 43, 101-107. https://doi.org/10.1249/MSS.0b013e3181e5797d

[37] Nguyen, L.L., Su, S. and Nguyen, H.T. (2014) Neural Network Approach for Non-Invasive Detection of Hyperglycemia Using Electrocardiographic Signals. Annual International Conference of the IEEE Engineering in Medicine and Biology Society, Chicago, 26-30 August 2014, 4475-4478.

[38] Zadeh, A.E., Khazaee, A. and Ranaee, V. (2010) Classification of the Electrocardiogram Signals Using Supervised Classifiers and Efficient Features. Computer Methods and Programs in Biomedicine, 99, 179-194.

https://doi.org/10.1016/j.cmpb.2010.04.013

Submit or recommend next manuscript to SCIRP and we will provide best service for you:

Accepting pre-submission inquiries through Email, Facebook, LinkedIn, Twitter, etc. A wide selection of journals (inclusive of 9 subjects, more than 200 journals)

Providing 24-hour high-quality service

User-friendly online submission system

Fair and swift peer-review system

Efficient typesetting and proofreading procedure

Display of the result of downloads and visits, as well as the number of cited articles

Maximum dissemination of your research work

Submit your manuscript at: http://papersubmission.scirp.org/

Or contact ti@scirp.org 